\title{
LA ESCRITURA DE JUVENTUD DE JUAN EMAR, PRELUDIO DE LA VANGUARDIA
}

\section{THE JUAN EMAR WRITING OF YOUTH, PRELUDE OF THE VANGUARD}

\author{
Sonia Rico Alonso. \\ Universidade da Coruña, España. \\ soniaricoalonso@gmail.com
}

\begin{abstract}
RESUMEN:
El escritor chileno Juan Emar (1893-1964) dejó un legado literario compuesto de sus obras publicadas en los años 30, Umbral y decenas de escritos inéditos en vida del autor que, en algunos casos, han visto la luz después de su muerte. En este trabajo queremos recuperar la escritura de juventud de Emar, aquella que elaboró entre 1910 y 1917 y a la que, en general, no se le ha reconocido valor. Mediante el estudio del cuaderno paradigmático de esa época (" $n$. 1", 1912-1914), y tomando como referencia la estética del autor, veremos cómo la escritura novel de Juan Emar constituye una muestra precoz, pero sólida, de ella, que enlaza de forma anticipada con técnicas y teorías propias del Emar que integró los movimientos de vanguardia a partir de la década de 1920.
\end{abstract}

PALABRAS CLAVE: Juan Emar, escritura de juventud, fragmento, fragmentarismo

\begin{abstract}
:
The Chilean writer Juan Emar (1893-1964) left a literary legacy composed of his works published in the 1930s, Umbral and dozens of unpublished writings throughout the author's lifetime. These have only been revealed after his death in some cases. In this work we want to recover Emar's youth writing, which he wrote between 1910 and 1917 and which, in general, has not been recognized its value. By studying the paradigmatic notebook of that time ("n. 1", 1912-1914), and taking the author's aesthetics as a reference, we will show how the novel writing of Juan Emar is an early, but solid sample of it. It connects in an early manner with techniques and theories typical of the avant-garde movements, of which Emar was parte from the 1920s.
\end{abstract}

KEY WORDS: Juan Emar, youth writing, fragment, fragmentary technique

Recibido: $22-05-20$

Aceptado: 09-11-20 
Sobre el escritor chileno Juan Emar, pseudónimo de Álvaro Yáñez (1893-1964), se ha afirmado a menudo que cuenta con una producción literaria extraña a la par que breve. La pública, desde luego, lo es: tres pequeños y originales libros editados en 1935 -Ayer, Miltín 1934 y Un año- y el volumen de cuentos Diez publicado dos años después. También es habitual añadir a este pequeño listado la obra gigante Umbral. Compuesta por más de 5.000 folios manuscritos, Emar comenzó a trabajar seriamente en ella hacia 1940 y siguió escribiéndola prácticamente hasta su muerte ${ }^{1}$ en 1964. Sin embargo, fuera de este conjunto de textos conocidos, escritos cuando el autor ya rondaba los cuarenta años y en adelante, hay una amplia y compleja producción que, en algunos casos, se ha ido revelando en los últimos años ${ }^{2}$.

Teniendo en cuenta toda la escritura del autor, deberíamos hablar, en realidad, de dos facetas: por una parte, la pública, integrada por los artículos de arte publicados en el periódico La Nación (1923-1927), Ayer, Miltín 1934 y Un año, de 1935, y un par de artículos de ese mismo año, y Diez (1937); y, por otra parte, la producción privada, compuesta por Umbral y "los papeles". Con este sintagma nos referimos a los documentos inéditos en vida de Emar: una inmensa cantidad de cuadernos y hojas de todos los formatos y de contenido heterogéneo, escritos a lo largo de toda su vida. Esta diversidad, su falta de clasificación y orden coherentes ${ }^{3}$ y el volumen del material han provocado que la crítica, en general, no haya penetrado en este valioso fondo, con algunas excepciones -Wallace 1997, Harris E. Schütte G. y Zegers B. 2006, Brodsky 1999 o Lizama 1998 y 2002-. Buena parte de él se compone, además, de escritos anteriores a la redacción de sus textos publicados, lo que demuestra que, aunque Emar tardó mucho tiempo en darse a conocer como escritor al

${ }^{1}$ Umbral no vio la luz de forma completa, sin embargo, hasta 1996, cuando fue publicada en cinco tomos por la Dirección de Bibliotecas Archivos y Museos (DIBAM) y el Centro de Investigaciones Diego Barros Arana. Previamente, en 1977, el editor argentino Carlos Lolhé había publicado el primer tomo de la obra.

${ }^{2}$ Nos referimos a algunos volúmenes con correspondencia privada del autor, a creaciones que Juan Emar descartó publicar y a diarios personales que en los últimos años han sido publicados. No obstante, buena parte del material escrito personal de Emar permanece inédito.

${ }^{3}$ El fondo bibliográfico del autor está repartido fundamentalmente entre dos lugares: el Archivo del escritor de la Biblioteca Nacional de Chile y la Fundación Juan Emar. En el primer caso, los diferentes cuadernos y folios sueltos están indexados en el catálogo de la Biblioteca Nacional y cada uno cuenta con una sencilla ficha descriptiva, aunque en algún caso la información aportada no es correcta o solo parcialmente. En cambio, la parte del fondo propiedad de la Fundación Juan Emar se encuentra más desordenada y no toda ella está indexada. Como hemos dicho, la cantidad del material es muy grande y se compone de muchas hojas sueltas y cuadernos -algunos en mal estado-, cualidades que dificultan enormemente la organización del fondo emariano. 
público, tenía una amplia experiencia como creador en el ámbito privado, experiencia que inició muy joven, siendo un adolescente. Es necesario, por tanto, revelar y analizar la producción aún desconocida o no estudiada a fondo del autor, dentro de la cual se encuentra su producción de juventud, que abarca el periodo entre 1910 y 1917 y de la que solo se posee una edición (2006), que merece, a nuestro juicio, una revisión ${ }^{4}$.

Álvaro Yáñez ${ }^{5}$ se inicia en la escritura en el año 1910 -cuenta con diecisiete años-, con la redacción de su texto "Mi estadía en Lausanne", primer escrito con carácter literario conocido y conservado del autor ${ }^{6}$, que consiste en la reescritura de unas notas tomadas durante su estancia de tres meses en la ciudad suiza de Lausanne en 1909. A partir de ahí, se conservan diez cuadernos posteriores -hasta $1917^{7}$-, la mayoría de ellos agrupados en dos series: por una parte, libretas dedicadas a la creación literaria y, por otra, libretas destinadas a ser diarios de vida. Fuera de estas series, se conserva otro cuaderno sobre viajes por Europa parecido al de Lausanne, un primer intento de narrativa de ficción de 1911 -el cuento "Torcuato"- y un cuaderno de 1912 muy heterogéneo, que se inicia como diario para terminar siendo un organigrama de la actividad creativa del autor por años, incluyendo también otro tipo de textos y dibujos. Dejando de lado la escritura diarística, interesante para otro tipo de estudios ${ }^{8}$, es el cartapacio "n. 1" (1912-1914) -el primero de la serie de libretas destinadas a la creación-, el cuaderno más representativo de la escritura de esta época, ya que es el más extenso y es fruto de la eclosión artística que sufre Álvaro Yáñez entre 1913 y 1914, cuando da inicio a buena parte de sus textos de juventud. Mediante su análisis revelaremos cómo funciona la escritura emariana de esta época, cuáles

${ }^{4}$ Esta edición constituye un primer paso en el proceso de revelación de estos escritos, ya que presenta un texto limpio, ordenado e inteligible. Sin embargo, el análisis concienzudo de cada libreta obliga a desplazar el foco de atención del texto propiamente al cuaderno y al paratexto. Ello obliga a redistribuir el corpus y a plantear una nueva teoría sobre él. Realizamos este estudio en nuestra tesis doctoral "La obra literaria de Juan Emar, Propuesta estética y arquitectura del texto" (2018).

${ }^{5}$ A lo largo de este trabajo emplearemos el nombre real del autor, ya que es con el que firma y se nombra a sí mismo en los textos. Su famoso pseudónimo no lo adopta hasta 1923, primero en francés -Jean Emar- y, a partir de 1926, en español -Juan Emar-, para firmar los artículos de arte que publica en La Nación.

${ }^{6}$ Anterior a este testimonio, solo se conserva un cuaderno escolar de 1905 que reutiliza hasta 1909 para hacer notas y dibujos carentes de valor literario.

71917 es el año de la última libreta conservada del primer periodo en la vida del autor, que él mismo establece en Umbral entre su nacimiento en 1893 y el fin de la Primera Guerra Mundial en 1918. Véase Emar, Umbral 74-76.

${ }^{8}$ Los diarios de juventud de Álvaro Yáñez son útiles para conocer no solo datos relevantes de su biografía (amistades, lecturas, viajes, etc.), sino también para estudiar el proceso de creación de sus textos y obras plásticas, que recoge por escrito en ellos. 
son los temas que le preocupan, qué estilo cultiva y qué vínculos se pueden establecer entre ella y sus creaciones posteriores.

Antes de comenzar, es necesario partir del hecho de que Álvaro Yáñez concibe de una manera muy original el soporte sobre el que escribe, en este caso, el cuaderno. En esta época, emplea diferentes tipos de libretas, en general, de un tamaño similar -el de libreta pequeña, más o menos un formato A5-, aunque con diferentes diseños externos y número de hojas. El uso corriente de un cuaderno consiste en escribir y numerar sus páginas de forma lineal desde la primera hoja hasta la última; sin embargo, Yáñez, de forma habitual, prescinde de la estructura lineal tradicional para presentar un texto fragmentado en pedazos que se distribuyen en diferentes partes del cuaderno y se alternan con trozos de otras creaciones.

El cuaderno paradigmático de Álvaro Yáñez en esta época contiene una especie de caos, retazos de textos que, a primera vista, parecen imposibles de seguir, sin conexiones aparentes los unos con los otros. Estos testimonios, además, presentan mucho texto residual - paratexto- en los márgenes de página o, incluso, entre líneas, que dificulta aún más su lectura. Sin embargo, son, precisamente, estos comentarios marginales los que guían al lector, pues, mediante ellos, el autor señala adónde se debe saltar para continuar cada texto, no solo dentro del propio cuaderno, sino también entre cuadernos 9 . De ahí que la numeración de las páginas de los cartapacios sea correlativa en cada una de las series temáticas existentes y no se limite a cada soporte.

Recapitulando, este anómalo modus operandi se advierte en buena parte de los testimonios conservados, sobre todo, en la serie de cartapacios con escritos literarios y en el ecléctico cuaderno de 1912. La serie de los diarios de vida, así como las dos libretas con escritos sobre viajes y el cuento "Torcuato" siguen, en general, una estructura lineal propia de la narrativa de ficción tradicional y del género del diario. Cabe decir, no obstante, que el propio género del diario constituye per se un texto fragmentario, al componerse de entradas independientes que recogen lo acontecido cada día.

\footnotetext{
${ }^{9}$ Yáñez hace muchos tipos de anotaciones en sus libretas. Las más importantes y frecuentes son, por un lado, indicaciones sobre los saltos en la lectura (ejemplo: “ $\rightarrow$ Pg. $81 \mathrm{MV}$ ”) y, por otro, unas marcas realizadas con un lápiz rojo que están relacionadas con Umbral, ya que Emar tomó algunos fragmentos de estos cuadernos para insertarlos - con más o menos adaptaciones- en su obra magna.
} 
Teniendo en cuenta la producción famosa y más estudiada del autor -la que publicó en los años 30 y Umbral-, no debería parecer extraño que el fragmentarismo sea el fenómeno característico de la escritura de Emar, pues es la técnica sobre la que se cimentan los textos emarianos y a la que dedicaremos más espacio hacia el final. No obstante, llama la atención especialmente en esta producción novel, ya que estos cuadernos eran absolutamente personales y su lectura quedaba restringida al propio Álvaro Yáñez. Así, esta especie de juego de cortes, pistas y saltos que constituye la escritura emariana de esta época parece un pasatiempo para su propio deleite, lo que acrecienta la excentricidad del autor, pero, como veremos, también está ligada a su original concepción del texto y la escritura.

Es el cuaderno “n. 1", redactado durante el periodo 1912-1914, aquel en que se advierte de forma ilustrativa esta singular metodología de trabajo. En este cartapacio se combinan dos tipos de escritos: por un lado, la anotación y el desarrollo de planes y proyectos, con borradores de textos, resúmenes de obras e indicaciones para sí mismo; y, por otro, las creaciones propiamente, es decir, la puesta en práctica de esos proyectos, concretamente, los textos "Ideas", "MV", "Diario de un solitario" y "Obs. Maup", que iremos viendo individualmente. Es un cuaderno, por lo tanto, fundamental porque recoge las primeras tentativas literarias del autor -el único texto literario conocido anterior es el cuento inconcluso "Torcuato" (1911) ${ }^{10}-\mathrm{y}$, además, refleja de un modo paradigmático el funcionamiento de la mente creativa de nuestro joven escritor, una mente completamente autorreflexiva, que encuentra en la escritura el espacio para conocer el mundo, que concibe tanto en su pluralidad como en su unidad, y para entenderse a sí mismo como ser que existe en él.

Como señalan Harris, Schütte y Zegers, Emar adolece de un problema, "el de la sobreabundancia” (12). Todas las obras de Emar provocan en el lector una sensación de rebosamiento, de desborde. Exceso de nombres, de cifras, de voces narrativas, de topónimos, de opiniones, de personajes, de acciones, de tipologías textuales, de imágenes, de tipografías...; sobreabundancia, en suma. Hay un afán en el escritor de contener en el texto la complejidad de la existencia -el problema de la pluralidad de la realidad- y este propósito nace prácticamente con él, pues sus escritos más tempranos ya plasman este

\footnotetext{
${ }^{10}$ Aunque afirmamos anteriormente que "Mi estadía en Lausanne" era el primer texto con carácter literario del autor, no deja de ser la reescritura en forma de narración de su diario de 1909. En cambio, "Torcuato" constituye el primer intento pleno de escritura de ficción, aunque guarde estrechos vínculos autobiográficos.
} 
modo de concebir la creación. El cuaderno "n. 1" es buena muestra de este fenómeno debido, sobre todo, porque es a finales de 1913 cuando Álvaro Yáñez se erige en escritor. De ahí que este cuaderno posea tal variedad de textos, que se complica cuando observamos la estructura externa de estos: en fragmentos dispuestos a lo largo de las páginas, alternándose unos con otros, y con los márgenes de página llenos de anotaciones. La escritura se entrecorta constantemente, como si el joven Yáñez no pudiera terminar un texto sin comenzar el siguiente, y así, el ansia por empezar la próxima creación supera cualquier cuestión formal, estructural o estilística. Por esta razón, desde bien joven el escritor no cree en la rigidez genérica ni en la correspondencia entre un soporte escritural destinado a un único texto. Para Emar no hay géneros, formatos o soportes, solo hay escritura, que brota de manera natural e impulsiva, tal y como entiende el mundo y la creación ${ }^{11}$.

Veamos más a fondo la forma y el contenido de "n. 1". Una vez pasada su tapa y su portada, comienza propiamente la escritura y el inicio de la paginación. Habrá que esperar, sin embargo hasta la página veintiuna de la libreta para hallar el comienzo de alguna de las creaciones literarias que nos permiten catalogar este cuaderno como el primero de esa serie de libretas. ¿Qué contienen, entonces, esas veinte primeras páginas? Textos variados y heterogéneos, como la enumeración y el desarrollo de proyectos - en estas páginas se diseña la estructura de algunas de las creaciones que vendrán a continuación-, brevísimos textos que parecen borradores o ensayos y un extenso y comentado resumen de algunos pasajes de la novela Resurrección (1899), de León Tolstói ${ }^{12}$. Esta primera parte del cuaderno resulta realmente compleja para su lectura, ya que los textos son breves y muy diferentes entre sí, y, además, algunos de ellos están fragmentados a lo largo de las veinte páginas iniciales ${ }^{13}$. No es, pues, hasta la página veintiuna cuando Álvaro Yáñez comienza su primer texto literario planeado y concebido como tal: "Ideas".

\footnotetext{
${ }^{11}$ Como veremos, su concepción está muy relacionada con la propuesta estética de los movimientos de vanguardia. El afán totalizador del artista provoca la percatación por parte del yo de la pluralidad de la realidad, que plasma en la obra de arte mediante la técnica fragmentaria y la mezcla de géneros.

${ }^{12}$ En la misma libreta, unas páginas después, también escribe otro resumen comentado, en este caso, de Historia de la civilización en Inglaterra (1857-1861), del historiador inglés Henry Thomas Buckle.

${ }^{13}$ La edición de los cuadernos de juventud de Álvaro Yáñez de 2006 recoge el contenido del cuaderno "n. 1", pero elimina todas estas partes que no son propiamente escritura literaria.
} 


\section{"Ideas" (finales de 1913 - 30 de junio de 1914 ${ }^{14}$ )}

El proyecto literario titulado "Ideas" está constituido por un conjunto de escritos de temática ecléctica; en realidad, una colección de opiniones y reflexiones sobre temas variopintos, algunos más trascendentales y otros más banales, como el amor, la moda, el arte, la ciencia, la voluntad, la guerra, el porvenir, etc.

"Ideas" representa, según sus editores, "un corpus de pensamiento en formación. [...] Ideas que el joven Álvaro Yáñez va ensayando sobre temas diversos, [...] a veces muy intuitivamente, otras de manera más ilustrada y elaborada" (Harris, Schütte y Zegers 1516). Son las reflexiones de un joven Álvaro Yáñez curioso e introvertido, inadaptado en su entorno familiar y social, que busca el trasfondo filosófico de todo lo que lo rodea y que, desde muy pronto, cuestiona todos los elementos que conforman su vida y la existencia en general. A modo de ejemplo, citamos un fragmento de una de las "Ideas" titulada "Simplificación":

Hay algo superior, diré, que gobierna pero no con la misma conciencia de los hombres sino con otra conciencia, otra inteligencia, otras miras, etc. que son las que se nos escapan. La ciencia constata sus efectos. El arte traduce la existencia de sensaciones inmateriales en nosotros y nat[urales] (Yáñez Bianchi 250).

En el cuaderno "n. 1" Emar escribe un total cuarenta y una ideas, tres en el año de 1913 y treinta y ocho en 1914, agrupadas en nueve bloques -entre ellos, se intercalan fragmentos de otras creaciones-. Cabe decir, que estas reflexiones continúan una vez que el cuaderno se finaliza en junio de 1914 en otro cuaderno de 1914 sin portada y, por tanto, sin título, que es la inmediata continuación del "n. 1"15.

\footnotetext{
${ }^{14}$ Esta fecha es la del fin del texto en el cuaderno "n. 1", si bien este continúa en otros cuadernos posteriores que no van a ser analizados aquí.

${ }^{15}$ También encontramos unas entradas llamadas "Ideas" en cuadernos posteriores a estos, de 1916 y 1917 "N. ${ }^{\circ}$ " y "N. ${ }^{\circ} 7$ "-, que podrían considerarse continuaciones de esta creación, ya que comparten el título. No obstante, se produce un salto temporal de casi dos años entre las "Ideas" de 1914 y las de 1916, lo que pondría en duda que se tratasen propiamente de una continuación -los editores responsables de la publicación de 2006 sí las consideran-. Además de la distancia temporal, hay una diferencia de forma: las "Ideas" originales, son textos, en general, breves, sobre asuntos muy variados, que se indican al comienzo de cada uno en una nota al
} 
Las "Ideas" de este cuaderno "n. 1" no surgen, o al menos no todas ellas, de manera espontánea en el joven escritor, sino que muchas nacen a raíz de conversaciones reales que mantiene a lo largo del día con sus amigos, familiares y conocidos. Ejemplo de ello son las "Ideas" "Importancia de Historia y Geografía” y "Algunas ideas sobre Religión”. Ambas poseen una nota marginal con una referencia a la lectura de su diario: "Ver Diario pag. 182" y "Ver Diario pag. 180"16, respectivamente. Efectivamente, si tomamos el diario correspondiente a 1913 y 1914 -“Cuaderno I. Diario”-, que escribía de forma paralela al cuaderno "n. 1" "17, comprobamos que el 31 de enero de 1914 Yáñez recoge: "En la comida discusión sobre conocimientos. Mis ideas sobre utilidad e influencia de la historia y geografía, ver cdno $N^{o} 2^{18}$, pág. 63" (Yáñez Bianchi 120); y el 28 de enero de ese mismo año: "En la noche discusión sobre religión. Mis ideas sobre religión: ver cuaderno $\mathrm{N}^{\mathrm{o}} 2$, pág. 68" (Yáñez Bianchi, 119). De modo que muchas de las "Ideas" que escribe Álvaro Yáñez surgen de conversaciones y acontecimientos de su día a día, en los que probablemente no expresaría su opinión, que reservaría para la seguridad y confianza que le proporcionaban sus libretas ${ }^{19}$.

En general, estos textos recogen meditaciones tempranas del autor, poco profundas en muchos casos, muy ligadas a los acontecimientos que vive en el día a día, con las que busca expresar su propio parecer y ahondar un poco más en los hechos. Otras ideas, sobre todo aquellas sobre la región superior -en términos intelectuales- o el amor son más complejas, ocupan más páginas y en ellas se advierten los intentos de un joven de diecinueve años que

margen junto a la fecha. En cambio, las "Ideas" de los cartapacios de 1916 y 1917 parecen un continuum, es decir, un único texto o entrada que se iniciaría el 29 de septiembre de 1916 y cuyo discurso continuaría a lo largo de varias fechas pasando incluso al cuaderno siguiente.

${ }^{16}$ Estas anotaciones carecen de referencia ya que no fueron incluidas en la edición de este cuaderno en la compilación de 2006. Solo se pueden leer, por tanto, en el manuscrito original.

${ }^{17}$ Como se puede observar, ambos cuadernos son nombrados como los primeros de una serie. Efectivamente, la libreta "n. 1", como hemos dicho, es la primera de la serie de libretas con textos literarios, mientras que "Cuaderno I. Diario" es el primero de la serie de los diarios. Para no confundir los cuadernos de una serie con los de la otra, Álvaro Yáñez emplea la numeración arábiga para la primera serie y la romana para la segunda.

${ }^{18}$ Puede resultar extraño que en estas referencias Álvaro Yáñez llame a su cuaderno "n. 1" "No 2". En realidad, el cuaderno que estamos estudiando fue llamando originalmente " $\mathrm{N}^{\mathrm{o}} 2$ ", pero en su portada lo tachó y lo sustituyó por "n. 1".

${ }^{19}$ La personalidad de Juan Emar lo llevó a crear un refugio en la escritura, un espacio reflexivo y privado para el autoconocimiento que fue construyendo desde la adolescencia. Los coetáneos a él destacan habitualmente su presencia silenciosa y su introversión. A modo de retrato del autor, citamos estas palabras de Volodia Teitelboim: "Invariablemente asistía a esas reuniones una pareja en que él callaba y ella hablaba. [...] Mirada y escuchaba todo con sus absortos, redondos ojos de lechuza, abiertos en la noche, como el pájaro dela sabiduría. Si en la larga velada decía una frase, carente de toda solemnidad, ella valía por toda la noche" (202). 
busca penetrar los elementos que componen la realidad en busca de su esencia, aunque ello implique, en ocasiones, un discurso un tanto farragoso y denso.

\section{"MV" (28 de diciembre de 1913 - 7 de julio de $1914^{20}$ )}

La segunda creación que Emar lleva a cabo en la libreta "n. 1" es el texto "MV", abreviatura del sintagma "Mi Vida". "MV" es un texto muy importante para su autor, al que dedica muchas menciones en otros de sus cuadernos, tanto en los diarios - "tendré también que avanzar algo, sea con M[i]V[ida] o cualquier otra cosa” (Yáñez Bianchi, 73)-, como en las listas de tareas que anota - " Hacer y concluir MV", "n. 1", diciembre de $1913^{21}$-. Por esta razón, así como por su importancia para con la propuesta estética y vital del autor, nos detendremos más en esta creación, a la que Álvaro Yáñez da comienzo el 28 de diciembre de $1913^{22}$, con dedicatoria incluida: "Al inolvidable recuerdo de Isoletta Cristinni"23. Este texto es el más largo de los que inicia en el cuaderno, junto a "Ideas", y lo estructura en seis bloques de escritura que dispersa a lo largo de la libreta.

"MV" constituye el intento inconcluso de una autobiografía redactada con veinte años. Desde luego, no es tanto el ego lo que impulsa al joven autor a emprender la tarea, sino que otros son los motivos principales, que él mismo enuncia al comienzo de esta:

Desde hace mucho tiempo he estado con deseos de escribir mi vida y para ello he llegado a acumular muchos datos que habrían podido servirme, pero no siempre he dejado en reposo a mis deseos pues no he encontrado el medio de cómo hacerlo. [...] Quiero escribir mi vida. ¿Para qué? Primeramente para darme el placer de leerla y releerla una vez concluida, placer que aumentará con los años, y enseguida para ver si soy capaz de darme cuenta, y de formular las causas que me han hecho obrar de tal o

\footnotetext{
${ }^{20}$ Esta fecha es la del fin del texto en el cuaderno "n. 1", si bien el texto continúa en otro cuaderno posterior.

${ }^{21}$ Esta nota aparece en la primera parte del cuaderno "n. 1", aquella que fue excluida de la edición, de ahí que carezca de referencia bibliográfica.

${ }^{22}$ Antes de esa fecha, como reflejan las citas anteriores, el texto ya estaba siendo planeado por su autor, que dedicó mucho tiempo a su diseño, tal y como refleja en su diario de 1913 ("Cuaderno I. Diario").

${ }^{23}$ Isoletta Cristinni fue una muchacha a la que Álvaro Yáñez conoció durante el viaje que realizó el autor junto a su madre -Rosalía Bianchi Tupper- entre América y Europa en mayo de 1912. Resulta curioso que Álvaro Yáñez le dedique un texto que comienza un año y medio después. No obstante, sabemos que, tras su separación, ambos mantuvieron un contacto epistolar y, de hecho, la dedicatoria de "MV" la ensaya ya en 1912 en el ecléctico cuaderno llamado inicialmente "Diario I", que redacta entre 1912 y 1917.
} 
cual forma, es decir, si soy capaz de averiguar el porqué de cuanto he hecho $[. .$.$] . Quiero estudiarme como un médico lo haría con un enfermo y$ toda la exquisita poesía que siempre rodea nuestro pasado, la dejaré sólo [sic] en mí mismo y llevaré al papel nada más que el fruto del raciocinio frío, sin pasiones (Yáñez Bianchi 131).

El texto lo concibe, pues, como un estudio de sí mismo, que permite ver ya cómo, desde bien temprano, Álvaro Yáñez se preocupa por trascender la realidad de las cosas. El hecho, asimismo, de que se trate de un estudio autobiográfico implica un desdoblamiento del yo en el sujeto escribiente y el sujeto sobre el que se escribe, fenómeno que entronca directamente con la teoría del desdoblamiento que, muchos años más tarde, Juan Emar sintetizaría en su artículo "Frente a los objetos" (1935):

Y luego..., luego me he vuelto a hojear a mí mismo, por un momento. Para ver si, cómo [sic] estoy ahora, soy o no soy así.

Hay muchos modos de hojearse, mas todos han de pasar por el desdoblamiento inicial: uno mismo que actúa; uno mismo que observa al que actúa. Al primero, échesele a meditar (y el segundo que vea cómo medita), échesele a estrellarse contras los afanes cotidianos, échesele a amar, échesele a odiar. El segundo, inmóvil (Emar cit. en Lizama, "Frente a los objetos" 139).

Vemos, pues, que aunque el autor no enunció su teoría de forma oficial hasta 1935, veintidós años antes ya tenía muy claro de qué forma concebía el mundo y el conocimiento que de este se puede tener. Así, como señala Lizama, la teoría del desdoblamiento no solo se enuncia en "Frente a los objetos" o se advierte en Umbral, sino "en el conjunto de la creación emariana" ("Frente a los objetos" 138). La precocidad de "MV”, por otro lado, da cuenta de la coherencia y madurez del proyecto escritural emariano, que parte de unas convicciones que establece muy joven y que desarrollará y sostendrá hasta el final de su vida.

Volviendo a "MV", si lo que trata de realizar es un análisis científico, tendrá que acotar y precisar el objeto de estudio, en este caso, su propia vida: 
Para facilitarme me dividiré mi vida en varios periodos y estos serán los siguientes: [I)] mi carácter, mis características en la niñez, [...] para ver si mi temperamento de hoy día tenía ayer sus raíces en el temperamento de ese niño [...]. II) [...] mis primeras aspiraciones que definieron mi carácter, aspiraciones de niño que pronto fracasaron y me dieron el primer dolor; III) Un primer amor que tanto influyó en mi modo de ser [...]. El viaje a Europa de 1909 y 1910 que abrió ante mi vista miles de horizontes nuevos [...] que nuevamente se derrumbaron en el IV periodo, o sea a la vuelta de Chile [...] V) Mi segundo y mayor amor, el que fue un tormento mientras existió, que me llenó el alma de pasiones atormentadas [...], y por último, el VI periodo mi segundo viaje a E[uropa] el olvido de ese amor, la calma que me vuelve, una aspiración ahora clara y firme que se alberga en mí, el formamiento [sic] de mi carácter definitivo y el comienzo de la marcha por el nuevo camino que le dio a mi vida (Yáñez Bianchi 131-132).

"MV" constituye un texto muy destacable en la producción novel de Juan Emar, no solo por la elección del género autobiográfico y lo que implica en la escritura emariana, sino porque gracias a él poseemos un conocimiento de primera mano de la percepción que tiene el autor de sí mismo, de su círculo más cercano -la burguesía liberal santiaguina- y de la sociedad de su país. La autobiografía comienza propiamente, y como había diseñado, con el periodo I, en que aporta una caracterización de su personalidad, sintetizada en la siguiente descripción:

Nací tímido y soñador. Siempre temblé ante una prueba de carácter; siempre pasé mis días muy lejos de la realidad que desde chico se me antojó antipática. [...] Y siempre, en el fondo, ha sido lo mismo: sueños y sueños que apenas llegaba a formular claramente y que solo tornaban tal o cual aspecto según las circunstancias que los hacían nacer (Yáñez Bianchi 134).

A la extensa descripción de su personalidad, sigue la caracterización del medio en que le ha tocado desarrollarse: su país -Chile, una "nación joven", como él mismo lo denomina - y su familia, ambos caracterizados por la moderación, el utilitarismo y la importancia de salvar las apariencias a ojos de los demás; actitudes que de ninguna manera concordaban con las del joven Álvaro Yáñez: 
Nací con un carácter incapaz de rebelarse ante las exigencias más pequeñas de $1 / 2^{24}$ y al mismo tiempo lleno de una pasión inextinguible que ardía en el fondo de mi alma y ese carácter tuvo que ceder y desarrollarse en una nación joven que necesariamente tenía como único fin las cosas prácticas y en una familia hija de la nación, una familia equilibrada, amante de las inteligencias tranquilas y de las vidas laboriosas, de una labor como la de todos. ¿Cuáles son los efectos de un temperamento así en un $1 / 2$ completamente opuesto? El relato de mi vida lo demostrará (Yáñez Bianchi 139).

Una vez descrito el carácter y el contexto de su nacimiento, Yáñez da pie a la narración de los primeros acontecimientos de su vida: la importancia de uno de sus tíos maternos, cuya muerte le causó una honda depresión, la relación con su hermana pequeña Inés -de muerte prematura- y con sus primos segundos Jorge y Marta Gaete o el sufrimiento experimentado al ingresar en el parvulario y en el colegio.

El segundo periodo de su vida lo inicia a mediados o finales de 1908, cuando es un adolescente de quince años que estudia en el Instituto Nacional. Es entonces cuando surge en él la idea de cambiar de vida: "no recuerdo cómo ni de qué modo, empecé poco a poco a pensar, a soñar más bien en la posibilidad de llevar otra vida o de siquiera adornar en algo la que llevaba" (Yáñez Bianchi 144). Se marca en este periodo objetivos intelectuales: leer, estudiar, meditar, escribir un diario, sacar fotografías, guardar recuerdos, hacer inventarios $^{25}$, etc. Comienza así a llenar cuadernos con proyectos -que nunca llega a completar-, que se multiplican conforme pasan los días y en cuyo desarrollo le otorga una importancia capital a su futuro viaje a Europa.

"MV" termina en el cuaderno " $n$. 1" al inicio del tercer periodo en la vida de Álvaro Yáñez, cuando experimenta el primer amor. La joven es Clara Pastor, de quien se enamora en el fundo familiar de Lo Herrera en una visita de ella y su hermana. Esta anécdota, escrita con cierto carácter naíf, debió de marcar al joven Álvaro Yáñez hasta tal punto que fue

${ }^{24}$ El autor emplea esta fracción para referirse al término medio, entendido como las circunstancias y condiciones externas que influyen en un individuo.

${ }^{25}$ La elaboración de listas e inventarios será una tarea constante en la trayectoria de Emar. De lo que trata, en definitiva, es de recoger todos los datos posibles sobre cualquier hecho o estado - de ahí también la redacción constante de diarios de vida-. Hay una obsesión permanente por no dejarse nada atrás para, después, darle forma y contenerlo en la obra literaria. 
recuperada muchos después en dos ocasiones por el autor ${ }^{26}$. Cabe decir, no obstante, que el texto "MV" no finaliza en el cuaderno "n. 1", sino que continúa, como "Ideas", en el siguiente, de 1914, alternándose fragmentos de ambas creaciones. Sin embargo, el joven escritor no finalizará su autobiografía, que abandonará ese mismo año, y así "MV" termina de forma extraña, con unos puntos suspensivos y con el relato truncado de su estancia en 1909 en la ciudad suiza de Lausanne. La nota marginal sugiere que el autor pensaba continuar; no obstante, nunca llegó a indicar la página a la que saltar para continuar la lectura. Por otra parte, y aunque los editores del texto colocan algunos fragmentos de otras libretas como continuaciones suyas ${ }^{27}$, todo parece indicar que "MV" fue abandonado en 1914 habiendo llegado en la autobiografía a los hechos de 1909. La prueba más evidente de ello, aparte de que no vuelve a haber menciones al texto en sus diarios, se encuentra una década después en el texto Amor (1923-1925), cuando el personaje de María le dice a Juan -alter ego del autor-:

Y ahora, m'hijito [sic], te voy a pedir una cosa: quiero que sigas escribiendo tu vida, que tienes tanto tiempo empezada y no sé por qué no terminas. Tal vez tú ahora miras con desprecio esa obra al concebir otras mejores. Si es así, hazlo entonces por mí, por tu Maluchita querida. No te puedes imaginar lo que me ha gustado lo poco que he leído y cuando pienso que ella se detiene a los 16 años, me da una pena muy grande. Síguela, pues, por mí. Así te conoceré más y querré a morir (Emar, Amor 177).

\section{"Diario de un Solitario" (6 - 22 de abril de 1914)}

Una vez finalizado el estudio de "MV" en este cuaderno y su continuación, nos quedan por tratar los dos últimos textos que Álvaro Yáñez comienza en la libreta "n. 1": "Diario de un Solitario" y "Obs. Maup.”. El primero de ellos es un diario ficticio de un

\footnotetext{
${ }^{26}$ El fragmento -con algunas modificaciones- fue retomado para el texto Amor (1923-1925) -pp. 43-45- y también lo insertará en el "Primer pilar" de Umbral -escrito después de 1940-, concretamente en la secuencia 52 (p. 534). Los casos de intertextualidad dentro de la propia obra de Juan Emar son muy frecuentes, de hecho, muchos fragmentos de los cuadernos de juventud, así como de las obras de los años 30, fueron incluidos en Umbral.

${ }^{27}$ Concretamente, entradas del diario que constituye el "Cuaderno IV. Diario" y de las libretas con escritos literarios "N. ${ }^{\circ} 5$ " y N. ${ }^{\circ} 6$ ". La propia lectura de los textos permite ver que no se trata de continuaciones, ya que los textos no guardan similitudes ni formales ni de contenido.
} 
personaje cuyo nombre desconocemos y el texto, al igual que "MV" e "Ideas", encuentra su continuación en la libreta siguiente.

Al comienzo del "Diario de un Solitario", y como ya había hecho al dar inicio a "MV", Álvaro Yáñez explica las razones que lo empujaron a llevar a cabo esta tarea, en este caso, la transcripción del diario personal del solitario:

\section{IV.914}

Voy a copiar a continuación un diario de un desconocido que presenta, al menos para mí, un gran interés, el de explicarnos, de hacernos comprender, más bien, uno de esos casos de locura que tan fríamente calificamos de incomprensibles y que, como se verá por este triste relato, obedecen a ciertas causas, es decir, no son un simple trastorno mental, sino la mente común de todo hombre dirigida hacia otros puntos que el de todos (Yáñez Bianchi 231).

Este diario ficcional, desarrollado en tres secuencias y cada una de ellas compuesta de varias entradas de diario $^{28}$, es calificado por sus editores como "si no uno de los primeros, el primer cuento con voluntad literaria de Álvaro Yáñez B.” (Harris, Schütte y Zegers 17). Desde luego, es uno de los primeros textos de ficción del autor, si bien consideramos que el cuento "Torcuato" (1911) constituye el primer texto con voluntad literaria del autor ${ }^{29}$. Se pueden establecer relaciones entre ambos textos $\mathrm{y}$, de hecho, guardan muchas similitudes al tratarse, fundamentalmente, de diarios de personajes de ficción. No obstante, si en "Torcuato" la hibridación discursiva era su característica central, es decir, la mezcla de géneros y voces discursivas, con prevalencia del diario, en "Diario de un Solitario" hay un único género definido -el diario- y una única voz -la del protagonista-, exceptuando el texto introductorio que hemos citamos anteriormente.

\footnotetext{
${ }^{28}$ Como hemos señalado, "Diario de un Solitario" continúa en el cuaderno inmediatamente posterior a "n. 1", carente de nombre. Su continuación también se compone de tres bloques de escritura dispersos por la libreta, cada uno de ellos integrado por varias entradas del diario ficcional.

29 "Torcuato" también integra la edición de textos de juventud publicada en 2006. Es posible que sus editores no lo consideren el primer texto literario del autor por el fuerte arraigo autobiográfico que posee -los protagonistas, Torcuato y Ofelia son alter ego literarios de Álvaro Yáñez y Marta Gaete, importante amor de juventud del autor-. Pese a ello, "Torcuato" posee muchos rasgos que lo caracterizan como texto de ficción, sobre todo, en cuestiones de técnica y estilo literarios que ya se atisban en él y que luego definirán la escritura madura de Juan Emar: la combinación de géneros literarios, las múltiples voces narrativas, el papel desempeñado por el yo protagonista, etc.
} 
Por otra parte, en la escritura emariana el yo es, en casi la totalidad de los casos y de forma declarada muchas veces, una figura identificable con el propio autor, esto es, con el propio Juan Emar -Álvaro Yáñez, en nuestro caso-; sin embargo, en el personaje del solitario no hay ninguna señal en el texto que nos indique quién es $y$, de hecho, aunque por algunos características -sentimiento de marginalidad y superioridad, rechazo a los que lo rodean, necesidad de soledad, aspiraciones intelectuales, etc.- podríamos relacionarlo con el propio autor, otros de sus rasgos - padres fallecidos, casa al borde del río, mención a nombres o iniciales de amigos, etc.- no concuerdan con los de Álvaro Yáñez. En cualquier caso, sí parece que, aunque el solitario fue creado como un personaje de ficción, el autor estampó muchas de sus aspiraciones, actitudes y sentimientos en la creación de este personaje, pues solo hace falta echar una ojeada a los diarios y reflexiones de Yáñez esos años para encontrar la misma manera de entender la vida, a los demás y a sí mismo. De hecho, en el texto introductorio que presenta el diario y que citamos al comienzo se advierte cierta actitud de justificación del modo de ser y actuar del personaje - no está loco, sino que su mente está enfocada en otros asuntos que no interesan a la mayoría-, que coincide con muchas de las reflexiones del propio Yáñez sobre sí mismo ${ }^{30}$.

\section{“Obs. Maup." (16 de abril - 3 de junio de 1914)}

Dicho esto, el último de los proyectos literarios que Álvaro Yáñez emprendió entre finales de 1913 y comienzos de 1914 es el texto “Obs. Maup.”. Se trata de un texto muy breve, compuesto de apenas tres secuencias y al que el autor decidió no dar continuidad fuera de este cartapacio. El extraño título de esta creación constituye una abreviatura del

\footnotetext{
${ }^{30}$ El 21 de julio de 1913 anota en su diario:
}

No sé por qué, desde algún tiempo tengo algo así como un débil presentimiento de que voy a concluir loco. Pienso en cuáles son mis ideas, cuáles mis tendencias y mis gustos, cómo y en qué sentido voy evolucionando y llego, muy a mi pesar, a ver que ese débil presentimiento, es una indiscutible realidad. Si alguien entrase en mí, ¿no vería en mi cerebro el cerebro de un loco? Pero hoy por hoy, mi cordura en mis actos, mi indiferencia en la vida, mi equilibrio en cuanto hago, demuestran que la locura está aún muy lejos. Desde luego, raro, soy! Ese odio al mundo es una rareza, común si se quiere, pero en mí la acompaña una tal impotencia de odiarlo, que sin duda, mi desprecio a la gente es un extravío de mi mente" (Yáñez Bianchi 66). 
sintagma "Observación Maupassant"31, ya que el escritor francés Guy de Maupassant constituía una de las lecturas habituales de Álvaro Yáñez en estos años ${ }^{32}$, que mantendría a lo largo de su vida y a la que dedicaría menciones y reflexiones en varios de sus textos personales. Teniendo en cuenta que Maupassant tiene la objetividad como principio estético y que para lograrla es imprescindible la observación minuciosa, con el título “Obs. Maup.”, el joven Yáñez nombra una serie de tres textos que constituyen análisis meticulosos de diversos asuntos al estilo de Guy de Maupassant. De hecho, en los textos iniciales de la libreta objeto de estudio, donde exponía proyectos a realizar, encontramos la mención "Poner en práctica observ. a lo Maup." y explica cómo llevarla a cabo.

En cuanto a su contenido, Harris, Schütte y Zegers lo califican como "una breve sección de textos introspectivos [...] que realiza el autor indagando en sí mismo, un tanto abisalmente, como en otra dimensión de las posibilidades de autoconocimiento y necesidad de trabajarse a sí mismo tanto en la vida como en la escritura” (18). A excepción de la segunda reflexión, donde sí se advierte bastante profundización, las otras analizan temas de la cotidianidad que carecen de relevancia fuera de lo puramente anecdótico. Así, en la primera observación el objeto observado es la pareja formada por Flora Yáñez, hermana del autor, y un joven entre los que se da una relación de amistad y coqueteo. Ellos representan a ojos de Álvaro Yáñez la frivolidad, la hipocresía, la superficialidad y el gusto por la apariencia y, por esta razón, critica la falta de profundidad de sus conocimientos y, sobre todo, el conformismo y la ingenuidad que los caracteriza.

La segunda observación resulta mucho más interesante, pues el objeto observado es el propio autor desde el distanciamiento, es decir, el autor se desdobla: uno -el narrador- es el que observa y otro -Pilo Yáñez ${ }^{33}$-, el observado. Se trata de un nuevo ejemplo de cómo la teoría del desdoblamiento la empieza a forjar y a poner en práctica desde sus primeras creaciones, como hizo también en "MV". En este caso, lo más destacable de esta entrada es

\footnotetext{
${ }^{31}$ Los editores del texto lo titulan "Obs. Manp"; sin embargo, el estudio minucioso de la caligrafía del autor y la argumentación sobre Guy de Maupassant que presentamos corroboran que la abreviatura es "Obs. Maup.".

${ }^{32}$ Por ejemplo, el 18 de agosto de 1913 anota en su diario:

Acabo de encontrar cuatro libros de Maupassant. ¡Qué hermoso! [...] ¡Oh, genio! Maupassant me hace vivir, es el estímulo más fuerte que he encontrado, mi cerebro entero se pone en ebullición cuando vislumbra esa alma doliente y sobrehumana, me hace el efecto que él con su mano alejara de mi lado todo lo vulgar del mundo, para mostrar en cambio todo lo enfermizo y grandioso que nos rodea (Yáñez Bianchi 75).

33 Álvaro Yáñez usa su apelativo familiar -Pilo- en el propio texto.
} 
la caracterización que Yáñez aporta de sí mismo como un ser ajeno a quien no logra comprender:

Pero no comprendo esos seres severos y graves que tienen dos pares de ojos: uno para juzgar a los demás y otro para ellos sin fijarse que si ellos se miraran con los reservados para los demás resultarían ellos iguales a los demás. ¡Pero no! Se creen perfectos porque los ojos que han hecho para verse los han hecho para que ellos resulten perfectos. P[ilo] es uno. $\mathrm{Su}$ característica mayor es la doble conciencia, apretada, severa, hasta testaruda para con los otros; amplia, grande, inmensamente elástica para él (Yáñez Bianchi 342).

Por último, la tercera y última observación está dedicada a la reflexión sobre la boda de un primo del autor, las fiestas en general y su comportamiento y el de su novia en tales eventos.

En definitiva, a excepción de la segunda entrada, que sí refleja un proceso de introspección y ahonda en la teoría del desdoblamiento, las otras inciden en mostrar a un Álvaro Yáñez inadaptado y frustrado ante un ambiente social con el que no se identifica y al que menosprecia e infravalora por carecer de aspiraciones intelectuales y artísticas.

Llegados a este punto, hemos visto cada uno de los textos literarios que Álvaro Yáñez inicia entre finales de 1913 y comienzos de 1914 y que constituyen, junto al cuento “Torcuato" (1911), sus primeras creaciones con vocación literaria plena. Es importante, no obstante, tener en cuenta en todo momento que estos textos se encuentran fragmentados y dispersos a lo largo del cuaderno "n. 1" y es este fenómeno el que nos interesa brevemente comentar.

\section{La escritura de juventud de Juan Emar y sus vínculos con la vanguardia}

Una vez presentada la libreta "n. l”, se advierte que el fragmentarismo es la técnica básica de composición de la escritura precoz del autor, como también lo será de sus obras maduras, tanto de las publicadas -Ayer, Miltín 1934, Un año y Diez-como de las inéditas en vida, como Amor, Cavilaciones o, por supuesto, Umbral. El fragmentarismo es también 
uno de los rasgos característicos de lo que se conoce como prosa de vanguardia ${ }^{34}$, en la que habitualmente se ha enmarcado la escritura de Juan Emar. Esta se define como aquellas manifestaciones en prosa, inclasificables si tenemos en cuenta los géneros tradicionales, realizadas en las primeras décadas del siglo XX, durante el desarrollo de los movimientos de vanguardia. Bajo este membrete es habitual encontrar a Juan Emar, como representante de la prosa de vanguardia en Hispanoamérica, junto a otros escritores como Macedonio Fernández, Felisberto Hernández, Vicente Huidobro, Martín Adán o Roberto Arlt, entre otros. Sus textos comparten, en general, una serie de características -empleo de la primera persona, reducción al mínimo o ausencia de trama, presencia de rasgos metaliterarios, mezcla de géneros, tipografías y lenguajes, uso experimental de la lengua, gusto por la parodia y el humor, etc. (Ferreiro 70-71) - que otorgan una mínima cohesión al conjunto y a la que contribuye también el empleo del fragmentarismo como técnica fundamental. Así, la estética de vanguardia, en cualquiera de sus lenguajes -literario, plástico, musical, etc.hace del fragmento unidad de conocimiento del mundo y, por extensión, unidad de composición de la obra. La obra, sin embargo, no consiste en la mera suma de fragmentos, sino que el todo resultante adquiere un significado nuevo, no equivalente a la suma de los significados de los pedazos que lo componen. La obra de arte se concibe, pues, desde una perspectiva holística ${ }^{35}$, es una realidad nueva, espacio para el conocimiento desde la óptica de un yo, que, precisamente por eso, constituye la voz principal del discurso y se priorizan géneros como la autobiografía o la autoficción -géneros que favorecen el desdoblamiento del sujeto, tan importante en Emar-.

Las obras de Juan Emar de los años treinta, así como Umbral, deben vislumbrarse desde esta óptica holística y, por eso, se componen de una serie de cuadros o episodios independientes -reflejo de la realidad perceptible y plural-y es el relato, es decir, el acto de contar, y no la historia, el que otorga coherencia a la obra. El aparente caos de las obras emarianas no es sino un reflejo de la complejidad del mundo, que pretende captar desde todos los ángulos posibles, en consonancia con la estética cubista -recuérdese el problema de la sobreabundancia del que hablamos al principio-. Las obras de Emar entroncan, por

\footnotetext{
${ }^{34}$ Sobre el fragmentarismo en la escritura de vanguardia, concretamente, sobre la técnica del collage, véase Yurkievich (1982).

${ }^{35}$ A este respecto, resultan muy esclarecedores los textos "De las posibilidades de la pintura" (1924), del pintor español cubista Juan Gris, y "Querer construir" (1930), del pintor uruguayo constructivista Joaquín Torres García.
} 
tanto, con la corriente vanguardista, y establecen vínculos con los textos de otros autores afines. Sin embargo, los cuadernos de juventud que hemos revelado, tomando como ejemplo el más paradigmático, son muy tempranos, de un periodo en que Emar aún no está familiarizado con la técnica literaria y, por supuesto, aún no ha tenido contacto con los círculos intelectuales parisinos, integrados por buena parte de los artistas de vanguardia tanto europeos -Guillaume Apollinaire, Antonin Artaud, Man Ray, Fernand Léger, etc.como hispanoamericanos -Vicente Huidobro, Acario Cotapos, Luis Vargas Rosas, los hermanos Ortiz de Zárate, etc.-. Álvaro Yáñez concibe, pues, la escritura desde una visión fragmentaria de la percepción del mundo, perspectiva que nace prácticamente con él, tal y como se aprecia en estos escritos noveles, que aparentemente solo constituyen un conjunto caótico de trozos de texto. Sin embargo, como se ha visto, es posible seguir los textos atendiendo a las indicaciones del autor y, por otro lado, el conjunto desordenado de pedazos textuales constituye no solo un divertimento para la lectura, sino que también plasma un modo muy particular de concebir la obra literaria, esto es, un modo holístico de entender el universo y su versión en miniatura, la obra de $\operatorname{arte}^{36}$.

En definitiva, parece razonable considerar que los textos de juventud de Juan Emar constituyen muestras literarias valiosas, que, aunque estilísticamente puedan revelar su falta de madurez, recogen ideas en las que el Emar adulto profundizará y desarrollará hasta establecer verdaderas teorías del conocimiento, de la existencia y del acto creativo. Asimismo, la estructura formal de estos cuadernos revela una concepción originalísima de la obra literaria, innata y adelantada a su contacto e integración a los movimientos de vanguardia. Por esta razón, es importante considerar esta producción novel como una muestra precoz de la escritura de Juan Emar, prueba fehaciente de la coherencia y magnitud de su proyecto escritural y, en definitiva, de su vida como escritor total.

\footnotetext{
${ }^{36}$ Lizama ha reflexionado al respecto:
}

Cada objeto o acontecimiento de la realidad es visto con una perspectiva orgánica, holística. El mundo se concibe como una unidad indivisible y dinámica cuyos elementos están estrechamente vinculados y pueden comprenderse sólo como modelos de un proceso cósmico. [...]

El atractivo de este modo de aprehender lo real está entonces en la convicción de que el universo constituye una compleja red de relaciones entre las diferentes partes de un conjunto unificado ("Emar y el deseo" 29). 


\section{BIBLIOGRAFÍA}

Brodsky, Pablo. "Introducción". Cartas a Carmen. Correspondencia entre Juan Emar y Carmen Yáñez (1957-1963). Juan Emar (autor). Santiago de Chile: Cuarto Propio, 1999. 9-13.

Emar, Juan. Umbral. Santiago de Chile: Dirección de Bibliotecas, Archivos y Museos (DIBAM) y Centro de Investigaciones Diego Barros Arana, 1996.

Emar, Juan. Amor. Santiago de Chile: La Pollera Ediciones, 2014.

Ferreiro, Carlos. "La prosa narrativa de vanguardia en Chile". Tesis doctoral. Universidade da Coruña. España, 2006.

Gris, Juan. "De las posibilidades de la pintura (1924)". Escritos de arte de vanguardia 1900/1945. Ángel González García, Francisco Calvo Serraller y Simón Marchán Fiz (eds.), (84-92). Madrid: Ediciones Istmo (1999). 84-92.

Harris E., Thomas, Daniela Schütte G. y Pedro Pablo Zegers B. "Introducción". M[i] v[ida]. Diarios (1911-1917). Álvaro Yáñez Bianchi (autor). Santiago de Chile: Dirección de Bibliotecas, Archivos y Museos (DIBAM) y LOM Ediciones, 2006. 719

Lizama, Patricio. “"Frente a los objetos:' fragmento de Juan Emar”. Taller de Letras 26 (1998): 137-141.

Lizama, Patricio. "Emar y el deseo de otra esencia para la vida". Paréntesis 8 (2001): 2533.

Lizama, Patricio. “Álvaro Yáñez/Jean Emar en Santiago de 1924”. Anales de Literatura Chilena 3 (2002): 223-226.

Rico Alonso, Sonia. "La obra literaria de Juan Emar. Propuesta estética y arquitectura del texto". Tesis doctoral. Universidade da Coruña. España: 2018.

Teitelboim, Volodia. (2002). "La noche del búho". Huidobro. La marcha infinita. Santiago de Chile: Editorial Sudamericana, 2002. 202-204.

Torres García, Joaquín. "Querer construir (1930)". Escritos de arte de vanguardia 1900/1945. Ángel González García, Francisco Calvo Serraller y Simón Marchán Fiz (eds.), (84-92). Madrid: Ediciones Istmo (1999). 277-279.

Wallace, David. "Proposiciones para la lectura de cuatro textos inéditos de Juan Emar". Tesis de magíster. Universidad de Chile. Chile: 1997.

Yáñez Bianchi, Álvaro. "n. 1". Cuaderno inédito, 1912-1914.

Yáñez Bianchi, Álvaro. M[i] v[ida]. Diarios (1911-1917). Eds. Thomas Harris E., Daniela Schütte G. y Pedro Pablo Zegers B. Santiago de Chile: Dirección de Bibliotecas, Archivos y Museos (DIBAM) y LOM Ediciones, 2006.

Yurkievich, Saúl. "Los avatares de la vanguardia". Revista Iberoamericana 48 (1982): 351366. 